INVESTIGACIÓN

https://doi.org/10.15198/seeci.2019.48.65-86

Recibido: 21/03/2017 --- Aceptado: 09/01/2019 --- Publicado: 15/03/2019

\title{
EL PRINCIPIO PRO HOMINE COMO BASE PARA LA LEGISLACIÓN DE MEDIDAS DE PROTECCIÓN DE GÉNERO
}

\section{THE PRO HOMINE PRINCIPLE AS A BASIS FOR THE LEGISLATION OF GENDER PROTECTION MEASURES}

Laura Victoria Córdova Pérez¹: Pontificia Universidad Católica del Ecuador Sede Ambato. Ecuador.

laura.cordova@uasb.edu.ec

Víctor Hugo Córdova Aldás: Universidad Técnica de Ambato. Ecuador. victorhcordova@uta.edu.ec

Héctor Fernando Gómez Alvarado: Universidad Técnica de Ambato. Ecuador hf.gomez@uta.edu.ec

\section{RESUMEN}

Las medidas de protección son aquellas actitudes y decisiones que toma en cuenta el Estado a través de sus diversas instituciones públicas, a fin de hacer efectivo el cuidado y protección de la víctima de la agresión, con respecto a la agresión misma y a su agresor; son mecanismos que buscan brindar apoyo y protección a las víctimas de las agresiones e impedir la continuación de estas. El presente trabajo de investigación, busca establecer la situación actual referente al otorgamiento de medidas de protección, así como la aplicación del principio Pro Homine al momento de la emisión de las mismas, como fundamento filosófico de la eliminación de la vulnerabilidad en género. Este trabajo muestra definiciones y aplicación del principio además de conclusiones y recomendaciones en la toma de decisiones bajo su dilucidación.

PALABRAS CLAVE: mujer - violencia - medidas de protección - principio Pro Homine.

\section{ABSTRACT}

The protection measures are those attitudes and decisions that the State takes into account through its various public institutions, in order to make effective the care

${ }^{1}$ Laura Victoria Córdova Pérez: Pontificia Universidad Católica del Ecuador, Sede Ambato. Abogada adjunta en libre ejercicio profesional para el Estudio de Asesoría Legal "ESDALEX", Abogada en libre ejercicio, Miembro de Jóvenes UNASUR Ecuador.

laura.cordova@uasb.edu.ec 
and protection of the victim of the aggression, with respect to the aggression itself and its aggressor; they are mechanisms that seek to provide support and protection to the victims of the aggressions and prevent their continuation. The present research work seeks to establish the current situation regarding the granting of protection measures, as well as the application of the Pro Homine principle at the moment of issuing them, as a philosophical basis for the elimination of vulnerability in gender. This work shows definitions and application of the principle as well as conclusions and recommendations in the decision making under its elucidation.

KEY WORDS: woman - violence - protective measures - principle Pro Homine.

\section{O PRINCÍPIO PRO HOMINE COMO BASE PARA A LEGISLAÇÃO DE MEDIDAS DE PROTEÇÃO DE GÊNERO}

\section{RESUME}

As medidas de proteção são aquelas atitudes e decisões que toma em conta 0 Estado através de suas diversas instituições públicas, afim de fazer efetivo o cuidado e proteção das vítimas de agressão, em relação a agressão e seu agressor; são mecanismos que buscam brindar apoio e proteção as vítimas das agressões e impedir a continuação das mesmas. $O$ presente trabalho de investigação, busca estabelecer a situação atual referente ao consentimento de medidas de proteção, assim como a aplicação do princípio Pro Homine ao momento da emissão das mesmas, como fundamento filosófico da eliminação da vulnerabilidade em gênero. Este trabalho mostra definições e aplicação do princípio ademais de conclusões e recomendações na toma de decisões em base a sua elucidação.

PALAVRAS CHAVE: mulher - violência - medidas de proteção - princípio Pro Homine.

\section{Cómo citar el artículo:}

Córdova P., L. V.; Córdova A., V. H., y Gómez A., H. F. (2019). El principio Pro Homine como base para la legislación de medidas de protección de género. [The Pro Homine principle as a basis for the legislation of gender protection measures]. Revista de Comunicación de la SEECI, 48, 65-86. doi: http://doi.org/10.15198/seeci.2019.48.65-86 Recuperado de http://www.seeci.net/revista/index.php/seeci/article/view/464

\section{INTRODUCCIÓN}

De acuerdo a lo que establece Montufar (1994, p. 15): "La violencia contra la mujer es una forma de actuación tan antigua como la más remota memoria histórica, y probablemente ha existido desde los primeros balbuceos de la sociabilidad", con lo establecido es claro que la violencia contra la mujer y es una actuación dentro de la sociedad, que se ha dado desde siempre, estableciendo un estatus de superioridad 
del hombre frente a la mujer dentro de la sociedad, tanto en aspectos públicos y privados. Ojeda, dice (2010, p. 88): "mientras el varón está obligado por la sociedad a trabajar en la esfera pública, la mujer tenía la responsabilidad "moral" de conservarse en la esfera privada, trabajar en los quehaceres de la casa y criar a los hijos", es claro que esta concepción se ha mantenido de generación en generación, se ha dado un sistema de roles marcados para cada género, el cual se ha inculcado en la educación que implanta modelos a seguir tanto para la mujer, en su rol de cuidar el hogar y a los hijos, es decir que su desempeño se encontraba en el ámbito privado del hogar. La problemática de la violencia contra la mujer y los demás miembros del núcleo familiar nace de cuestiones antropológicas, no meramente de cuestiones sociales o culturales, ya que desde tiempos antiguos se ha establecido estos roles para cada sexo, tanto hembra como macho, desde épocas prehistóricas en las que el hombre debía salir a buscar el alimento y la mujer quedarse al cuidado del hogar y de la familia, todo esto ha sido un pauta para que cada cultura haya establecido este comportamiento como un parámetro obligatorio dentro de la sociedad. Con la entrada en vigencia el nuevo (Código Orgánico Integral Penal, Registro Oficial: No 180, 2014), la violencia física como psicológica hacia la mujer y miembros del núcleo familiar se estableció como un delito de acción pública contemplado en el artículo 155 del Código antes mencionado. Es un cambio dentro del ordenamiento jurídico, que ha presentado dificultades de procedimiento en cuanto a la correcta y efectiva aplicación de las medidas de protección para las víctimas de estos delitos. Con el Código Penal anterior, este tipo de agresiones eran tramitados dentro de la Unidad de Violencia contra la Mujer y la Familia, institución donde al receptar denuncias con relación a violencia contra la mujer, se emitía de manera inmediata medidas de protección a la víctima, con el objetivo de preservar su integridad y protegerla de futuras agresiones.

Dentro de la Constitución de la República del Ecuador se establecen los principios del ejercicio de los derechos, especificando que en materia de derechos y garantías constitucionales, las servidoras y servidores públicos, administrativos o judiciales, deberán aplicar la norma. Esto en concordancia directa con lo que establece el precepto del principio Pro Homine mismo que inquiere que la interpretación jurídica busque el mayor beneficio para la persona, es decir, que debe acudirse a la norma más amplia o a la interpretación extensiva cuando se trata de derechos protegidos, con prioridad si implica el beneficio a la integridad, seguridad y protección de la víctima. Es en base a lo expuesto se pone en cuestión la efectividad de los procesos judiciales en materia de delitos de violencia contra la mujer y miembros del núcleo familiar, específicamente en el otorgamiento oportuno de medidas de protección, las mismas que no se establecen de manera inmediata y hasta puede ser negado si no se cuenta con elementos que motiven el proporcionar las mismas, lo cual deja a las víctimas de este tipo de delitos en un estado de inseguridad e indefensión, aumentando el riesgo de ser sujeto de agresión nuevamente o a su vez ser víctima de un delito más grave. Este problema se origina cuando al presentarse una denuncia por agresión en contra de la Mujer o miembros del núcleo familiar, el fiscal debe solicitar al juez el otorgamiento de las medidas de protección las cuales deben tener fundamentos valederos para otorgarlas, lo cual hace que las medidas de protección no sean inmediatas. Esto se origina en la normativa la cual determina el 
Córdova P., L. V.; Córdova A., V. H., y Gómez A., H. F. El principio Pro Homine como base para la legislación de medidas de protección de género

procedimiento a tomar dentro de los casos de violencia contra la mujer o miembros del núcleo familiar, que establece que debe ser motivado el otorgamiento de medidas de protección, tanto en la solicitud del fiscal como en el otorgamiento del Juez.

Este trabajo constituye un proyecto de investigación referente al otorgamiento de medidas de protección en los delitos de violencia contra la mujer y miembros del núcleo familiar, debido a que dentro de la legislación ecuatoriana se ha dado un cambio al trámite a seguirse en estos casos, puesto que actualmente se encuentra tipificado como un delito, por tanto es un proceso que debe ser tramitado por Fiscalía y ha diferido del procedimiento que se seguía con la ley anterior, específicamente en cuanto al otorgamiento de medidas de protección. La finalidad de esta investigación está en conocer cómo afecta este tipo de dilaciones a las víctimas de este tipo de violencia, así también el conocer como pone en riesgo su integridad y sobretodo establecer con claridad cuáles son las medidas de protección aplicables a estos casos y de qué manera estas podrían ser más efectivas en cuanto a su otorgamiento. Para dejar clara nuestra propuesta, en el Capítulo I, Fundamentos Teóricos, encontramos el estado de la cuestión, que es un repaso de las investigaciones previas con relación a la temática; seguido de la descripción del problema planteado, que es la exposición de las causas y consecuencias de la investigación; a su vez la exposición de las preguntas básicas que colaboran a comprender el problema; posteriormente están los objetivos tanto general como específicos, que son las metas a conseguir dentro de la investigación; se señala a su vez la pregunta de estudio, que es el resultado de la investigación. Tenemos también el señalamiento de variables, ya que interviene una relación de causa-efecto; después la red de inclusiones conceptuales y finalmente los fundamentos teóricos, en donde se desarrolla los temas y subtemas referentes a la investigación.

En el Capítulo II, Metodología, se describe la metodología de la investigación, en donde se identifica el enfoque, la modalidad y tipos de investigación empleados en el desarrollo del trabajo, las fuentes de investigación y las técnicas e instrumentos utilizados, para poder lograr los objetivos planteados anteriormente. En el Capítulo III, Resultados, se encuentra el análisis y la interpretación de los resultados, se establece un análisis detallado de aquello que recibimos como derivación y como respuestas a la aplicación de los instrumentos de investigación. Finalmente Conclusiones y Recomendaciones, que surgieron a partir del presente proyecto de investigación afianzando la importancia del mismo.

En cuanto a esto (Chávez, 2012) establece que este tipo de violencia no se puede abordar como un problema individual o acto aislado, considerando las circunstancias particulares entre el agresor y la víctima sino como una problemática social que ancla sus raíces en las relaciones sociales de desigualdad. Dentro de la investigación de (Quiña, 2010) desarrollada en el cantón Ambato se establece que un gran porcentaje de personas conocen la definición de Violencia Intrafamiliar pero casi el mismo porcentaje de personas desconoce sobre las medidas de protección contra estas agresiones, así como quienes han sido beneficiadas con medidas de protección no han hecho uso de estas, lo que ha producido la reincidencia de violencia. Esto en 
concordancia directa con lo que nos establece la investigación desarrollada en el cantón Ambato de (Quinatoa, 2012), quien concluye que existe un desconocimiento de las medidas de amparo por parte de la población, lo que impide un correcto ejercicio de la administración de justicia, desembocando en nuevos casos de violencia intrafamiliar.

Es claro que la violencia que existe dentro de un hogar es un acto consciente que se manifiesta de un miembro a otro, siempre estos parámetros de desigualdad se han establecido dentro de los lineamientos de la sociedad, indicando que siempre va a existir una figura de superioridad y a su vez los demás miembros deben mantener una posición de subordinación, esta jerarquía se encuentra definida como se expuso anteriormente, por factores de género y edad. Para poder tener una explicación más clara de cómo opera el agresor sobre las víctimas, es importante citar lo que establece (Walker, 1979), a decir de esta autora cuando existe una relación de violencia, la mujer se encuentra dentro de un ciclo el cual consta de tres fases: 1) la fase de acumulación de tensión, 2) la fase de explotación violenta y 3) la de luna de miel. Cada una de ellas tiene características específicas que marcan una relación violenta.

1-La fase de tensión: Se caracteriza por el incremento de tensión y da comienzo a los incovenientes en la pareja.

El hombre violento expresa hostilidad, pero no en gran magnitud. La mujer intenta calmar, complacer o, no hacer lo que pueda molestar a su pareja, teniendo la creencia ilusoria que ella puede controlar la agresión.

3- Fase de conciliación: Esta fase es conocida como luna de miel en la que el hombre violento presenta arrepentimiento, le hace promesas de cambio o le hace regalos.

Esta fase es un estimulo positivo para que la mujer mantenga la relación. Con el que ve el lado positivo de su pareja, por lo cual asume que puede cambiar.

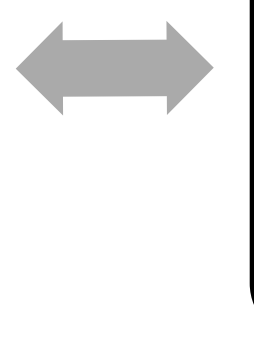

2- Fase de agresión: En esta fase se manifiesta la violencia psíquica, física y/o sexual.

La mujer suele denunciar los malos tratos y en la que puede decidirse a contar lo que está pasando.

Gráfico 1. Ciclo de la violencia. Fuente: elaboración propia. 
Córdova P., L. V.; Córdova A., V. H., y Gómez A., H. F. El principio Pro Homine como base para la legislación de medidas de protección de género

Este ciclo de violencia explica claramente como la violencia cumple un ciclo el cual con el tiempo presenta ciertos cambios, la fase de agresión se repita más a menudo o se mantiene todo el tiempo entre la tensión y la agresión, escasamente pasa por la fase de conciliación, lo que implica en nivel de violencia más alto. Es importante destacar que durante dos fases de este ciclo la persona violentada soporta dicha violencia con la expectativa de cambio de su pareja o a su vez al mostrar arrepentimiento y una buena actitud, impide que la víctima tenga una decisión clara de cesar con la violencia.

Como hemos podido indicar en el ciclo de violencia, esta es tomada como una dinámica normal, en la cual en la mayoría de casos quien es la victima de las agresiones, siente responsabilidad que se hayan producido las mismas y siente que debe tomar una actitud de sumisión para no provocar una futura agresión.

Al hablar de violencia tanto de género como intrafamiliar, es claro que es una problemática de importancia tratada a nivel global, lo cual ha llegado a generar una concientización general, que por medio de esfuerzos conjuntos ha sido calificada como de interés colectivo. Gracias a este proceso de concientización el cual ha nacido de algunos movimientos de lucha y defensa de derechos, se ha alcanzado reconocimiento dentro de la comunidad internacional, estos esfuerzos se han podido plasmar dentro de tratados y convenios internacionales que hoy en día tienen rango constitucional e incluso supraconstitucional.

La evolución que ha tenido los derechos humanos en general y a su vez específicamente en relación a la temática de violencia de género e intrafamiliar se puede ver reflejada en el siguiente cuadro:

Tabla 1. Evolución de derechos en violencia de género e intrafamiliar a nivel internacional.

\begin{tabular}{|c|c|c|c|}
\hline Organismo & Fecha & Normativa & Avance \\
\hline $\begin{array}{l}\text { Organización de } \\
\text { las Naciones } \\
\text { Unidas (ONU) }\end{array}$ & 1945 & Carta fundacional & $\begin{array}{l}\text { Resuelve corroborar la } \\
\text { confianza en los derechos } \\
\text { fundamentales del hombre, } \\
\text { el valor de la persona } \\
\text { humana y en la igualdad de } \\
\text { derechos de hombres y } \\
\text { mujeres. }\end{array}$ \\
\hline $\begin{array}{c}\text { Asamblea General } \\
\text { de la ONU }\end{array}$ & $\begin{array}{c}10 \text { de diciembre de } \\
1948\end{array}$ & $\begin{array}{c}\text { Declaración Universal } \\
\text { de los Derechos } \\
\text { Humanos }\end{array}$ & $\begin{array}{l}\text { Por medio de estos treinta } \\
\text { artículos se promueve el } \\
\text { reconocimiento de libertades } \\
\text { y derechos fundamentales } \\
\text { de todos los seres humanos, } \\
\text { en defensa de la igualdad de } \\
\text { las personas. }\end{array}$ \\
\hline $\begin{array}{c}\text { Asamblea General } \\
\text { de la ONU }\end{array}$ & Años 70 & $\begin{array}{l}\text { Convención para la } \\
\text { eliminación de todas } \\
\text { las formas de } \\
\text { discriminación contra la } \\
\text { mujer (CEDAW) }\end{array}$ & $\begin{array}{l}\text { Se aprueba la creación del } \\
\text { Comité para la eliminación } \\
\text { de la discriminación contra } \\
\text { la mujer, órgano encargado } \\
\text { de supervisar la aplicación }\end{array}$ \\
\hline
\end{tabular}


Córdova P., L. V.; Córdova A., V. H., y Gómez A., H. F. El principio Pro Homine como base para la legislación de medidas de protección de género

\begin{tabular}{|c|c|c|c|}
\hline & & & $\begin{array}{l}\text { de esta declaración, el cual } \\
\text { más completo instrumento } \\
\text { jurídico internacional de } \\
\text { derechos humanos para las } \\
\text { mujeres, a fin de alcanzar } \\
\text { una igualdad de condiciones } \\
\text { entre hombres y mujeres. }\end{array}$ \\
\hline $\begin{array}{c}\text { ONU } \\
\text { Estados Miembros }\end{array}$ & 1975 & $\begin{array}{c}\text { Primera Conferencia } \\
\text { Mundial de la Mujer } \\
\text { (México) }\end{array}$ & $\begin{array}{l}\text { Solicita a los Estados } \\
\text { Miembros asumir } \\
\text { compromisos concretos para } \\
\text { desarrollar estrategias y } \\
\text { planes de acción con el } \\
\text { objetivo de erradicar la } \\
\text { discriminación, logrando } \\
\text { igualdad e integración de las } \\
\text { mujeres en todas las áreas } \\
\text { de la sociedad. }\end{array}$ \\
\hline $\begin{array}{c}\text { ONU } \\
\text { Estados Miembros }\end{array}$ & 1980 & $\begin{array}{c}\text { Segunda Conferencia } \\
\text { Mundial sobre la Mujer } \\
\text { (Copenhague) }\end{array}$ & $\begin{array}{l}\text { Se realiza una evaluación del } \\
\text { plan de acción mundial } \\
\text { propuesto cinco años atrás } \\
\text { en la primera conferencia. } \\
\text { Se establece que la violencia } \\
\text { contra las mujeres, } \\
\text { incluyendo la violencia } \\
\text { doméstica, constituye una } \\
\text { violación a sus derechos } \\
\text { humanos y consta como } \\
\text { asunto de orden público. } \\
\text { Antes de la conferencia, el } \\
\text { tema se trataba } \\
\text { generalmente como un } \\
\text { asunto del ámbito privado } \\
\text { donde el Estado no podía } \\
\text { intervenir. }\end{array}$ \\
\hline $\begin{array}{c}\text { ONU } \\
\text { Estados Miembros }\end{array}$ & 1985 & $\begin{array}{l}\text { Conferencia Mundial } \\
\text { para el Examen y la } \\
\text { Evaluación de los } \\
\text { logros del Decenio de } \\
\text { Naciones Unidas para } \\
\text { la Mujer: Igualdad, } \\
\text { Desarrollo y Paz } \\
\text { (Nairobi) }\end{array}$ & $\begin{array}{l}\text { Se concluye que las mejoras } \\
\text { en la situación jurídica y } \\
\text { social de las mujeres y los } \\
\text { esfuerzos para reducir la } \\
\text { discriminación que se habín } \\
\text { alcanzado no eran } \\
\text { suficientes y habían } \\
\text { beneficiado únicamente a } \\
\text { una minoría de mujeres. } \\
\text { Se plantean nuevos } \\
\text { lineamientos para obtener } \\
\text { mejores resultados antes del } \\
\text { año } 2000 . \\
\text { Se concluye que la violencia } \\
\text { hacia las mujeres, } \\
\text { particularmente la violencia } \\
\text { doméstica, es un problema } \\
\text { amplio y en aumento y }\end{array}$ \\
\hline
\end{tabular}


Córdova P., L. V.; Córdova A., V. H., y Gómez A., H. F. El principio Pro Homine como base para la legislación de medidas de protección de género

\begin{tabular}{|c|c|c|c|}
\hline & & & $\begin{array}{l}\text { representa una limitación } \\
\text { para la equidad entre los } \\
\text { géneros y una ofensa a la } \\
\text { dignidad humana } \\
\text { Se solicita a los gobiernos } \\
\text { establecer programas y } \\
\text { medidas que permitan a las } \\
\text { mujeres el acceso a medios } \\
\text { de defensa efectivos. }\end{array}$ \\
\hline $\begin{array}{c}\text { ONU } \\
\text { Estados Miembros }\end{array}$ & 1993 & $\begin{array}{c}\text { Segunda Conferencia } \\
\text { Mundial de los } \\
\text { Derechos Humanos }\end{array}$ & $\begin{array}{l}\text { Se reconoce expresamente, } \\
\text { que los derechos humanos } \\
\text { de la mujer y de la niña son } \\
\text { parte inalienable, integrante } \\
\text { e indivisible de los derechos } \\
\text { humanos universales. }\end{array}$ \\
\hline $\begin{array}{c}\text { Asamblea General } \\
\text { de la Organización } \\
\text { de Estados } \\
\text { Americanos (OEA) }\end{array}$ & 1994 & $\begin{array}{c}\text { Convención } \\
\text { Interamericana para } \\
\text { prevenir, sancionar y } \\
\text { erradicar la Violencia } \\
\text { contra la Mujer } \\
\text { "Convención de Belèm } \\
\text { Do Pará" }\end{array}$ & $\begin{array}{l}\text { Este instrumento jurídico } \\
\text { internacional es de gran } \\
\text { importancia ya que posee } \\
\text { carácter vinculante y } \\
\text { obligatorio ante los } \\
\text { gobiernos de los Estados } \\
\text { miembros para asumir } \\
\text { medidas de acción y a su } \\
\text { vez registrar y evaluar la } \\
\text { efectividad de la aplicación } \\
\text { de las mismas. }\end{array}$ \\
\hline $\begin{array}{c}\text { ONU } \\
\text { Estados Miembros }\end{array}$ & 1995 & $\begin{array}{c}\text { Cuarta Conferencia } \\
\text { (Beijin) }\end{array}$ & $\begin{array}{l}\text { Se aprueba la Declaración y } \\
\text { Plataforma de Acción de } \\
\text { Beijing, en la misma se } \\
\text { plantean como objetivos la } \\
\text { erradicación de la } \\
\text { discriminación a la mujer por } \\
\text { diferentes aspectos. }\end{array}$ \\
\hline
\end{tabular}

Fuente: elaboración propia.

\subsection{La violencia de género e intrafamiliar desde la perspectiva nacional}

Como se ha podido establecer la violencia de género es un problema global, el cual no es ajeno a la realidad de nuestro país, que como conocemos también ha sido susceptible a cambios y evoluciones en cuanto a derechos y protección de los mismos, para lo cual es importante establecer los hechos más relevantes que se han desarrollado en el país a lo largo de la historia en cuanto a la temática central de esta investigación.

Para poder tener un punto de partida podemos establecer el inicio del gobierno de Jaime Roldós Aguilera, en este mandato se dio más importancia a la temática de vulneración de derechos, pero todavía no enfocada en cuanto a violencia de género.

Uno de los cambios más notorios se pudo apreciar a partir de los años 80 , se toma en cuenta la diferencia entre géneros, a su vez nace la concientización en cuanto al tema de la violencia contra la mujer, por parte de ciertas agrupaciones. 
Ya en los años 90, la presión social que se ejerció por medio de organizaciones feministas, dio pasó a que se establezcan proyectos en cuanto a políticas públicas con enfoque a la eliminación de todo tipo de violencia ya sea contra la mujer o intrafamiliar, desde este punto han iniciado hechos de mayor relevancia en cuanto al progreso en este tema, de los cuales podemos establecer algunos:

El primer organismo en crearse en pro de la mujer fue la Dirección Nacional de la Mujer (DINAMU), el cual formaba parte del Ministerio de Bienestar Social, este se convirtió en el Consejo Nacional de las Mujeres (CONAMU), a partir del año de 1997, como consta dentro del Registro Oficial No. 128, esta entidad se crea a partir del compromiso pactado en la Conferencia de la Mujer de Beijín, esta entidad tenía como finalidad el fomentar políticas públicas en beneficio de la igualdad de género y el respeto de los derechos de las mujeres, en esta época nacieron también otros organismos los cuales establecieron los primeros consultorios jurídicos gratuitos para llevar temas de violencia de género e intrafamiliar específicamente. Es importante señalar que la violencia de género e intrafamiliar, no era considerada como un problema de carácter público, lo cual al ser únicamente un asunto de perfil privado, se tornaba más difícil para su tratamiento y la seguridad de las víctimas.

En el año de 1994, inicia la creación de una de las instituciones más relevantes en materia de violencia de género e intrafamiliar, que son las Comisarías de la Mujer y la Familia, pero su campo de acción se vio limitado ya que carecían de fundamento legal para la tutela de justicia en materia de violencia de género e intrafamiliar, de manera que no existían acciones legales efectivas que pudieran ser dispuestas.

Ya para el año de 1995, se aprueba la primera ley para poder dar juzgamiento a delitos de violencia de género e intrafamiliar, la cual fue aprobada el 11 de diciembre, dentro del Registro Oficial No. 839, la Ley Contra la Violencia a la Mujer y la Familia, también conocida como Ley 103, la misma que estableció a la violencia intrafamiliar como una infracción, por ende se disponía sanciones y mecanismos de protección en favor de las víctimas. Esta ley era ejecutada por medio de las Comisarias de la Mujer, anteriormente ya constituidas.

Después de tres años surge otro importante avance dentro de la Constitución Política del Ecuador (1998), dentro de la cual se establece que el Estado se encuentra obligado a tomar medidas para prevenir, eliminar y sancionar, la violencia contra los niños, adolescentes, mujeres y personas de la tercera edad.

En 2008, con la puesta en vigencia de la actual Constitución de la República, se da reconocimiento a la integridad personal, en todos los aspectos tanto público como privado. Por lo cual en el caso de violencia intrafamiliar y de género, se garantiza atención prioritaria y especializada tanto para con la víctima, como para el juzgamiento, en virtud de esto el Estado adopta medidas necesarias para prevenir, eliminar y sancionar toda forma de violencia, en especial la ejercida contra las mujeres y el núcleo familiar. Para la ejecución de todo lo expuesto dentro del Código Orgánico de la Función Judicial (2009), se dispone que la potestad jurisdiccional se ejercerá por las juezas y jueces en forma especializada, según las diferentes áreas de la competencia; a su vez se indica que en los casos de violencia de genero e intrafamiliar, no cabe la mediación ni el arbitraje y se crean los Juzgados de Violencia contra la Mujer y la Familia. 
Para el año 2013, recién se dio la posesión de los jueces especializados en la materia de violencia, con lo cual se dio inicio al trabajo de las Unidades Judiciales contra la Violencia a la Mujer o Miembros del Núcleo Familiar.

En el año 2014, con la puesta en vigencia del Código Orgánico Integral Penal, en Registro Oficial No 180, la temática de la violencia de género e intrafamiliar, toma una nueva dirección, ya que la violencia deja de considerarse únicamente como una contravención, para ser tipificada como delito, por ende el órgano encargado de su procedimiento pasa a ser la Fiscalía, con juzgamiento en sede Penal.

Como hemos podido constatar la violencia de género e intrafamiliar ha sido un problema de trascendencia dentro de nuestro país, el mismo que ha tenido una evolución un poco tardía, pero la cual en los últimos años ha tenido muchos proyectos y esfuerzos para cambiar su situación.

\subsection{La violencia de género e intrafamiliar desde la perspectiva provincial}

Al hablar de la problemática sobre violencia de género e intrafamiliar, es importante destacar la situación actual que enmarca a nuestra provincia. Por lo cual la situación de la misma se podrá establecer por medio de gráficos.

El Instituto Nacional de Estadísticas y Censos - INEC (2011), estableció la Primera Encuesta Nacional de Relaciones Familiares y Violencia de Género contra las Mujeres - Tungurahua, la cual fue una respuesta a la necesidad de información sobre nuestro medio enfocada únicamente al tema de violencia de genero e intrafamiliar del que se desprende la siguiente información:

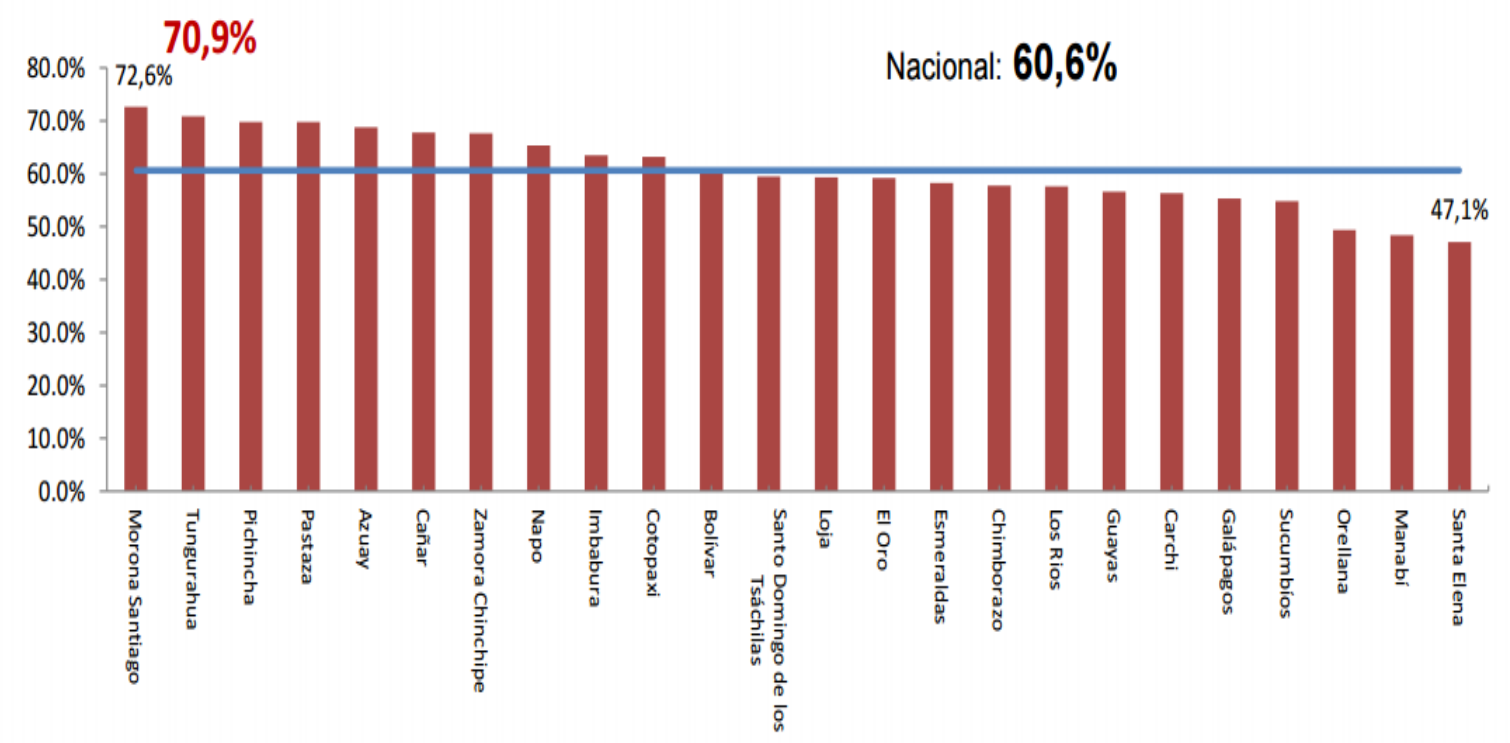

Gráfico 2. Mujeres que han vivido algún tipo de violencia - Por provincia.

Fuente: Primera Encuesta Nacional de Relaciones Familiares y Violencia de Género contra las Mujeres - Tungurahua. 
De acuerdo a la encuesta realizada, Tungurahua es la segunda provincia que registra mayor violencia contra las mujeres con $70,9 \%$ frente al $60,6 \%$ de mujeres a nivel nacional. Considerando todos los tipos de violencia: física, psicológica, sexual, patrimonial.

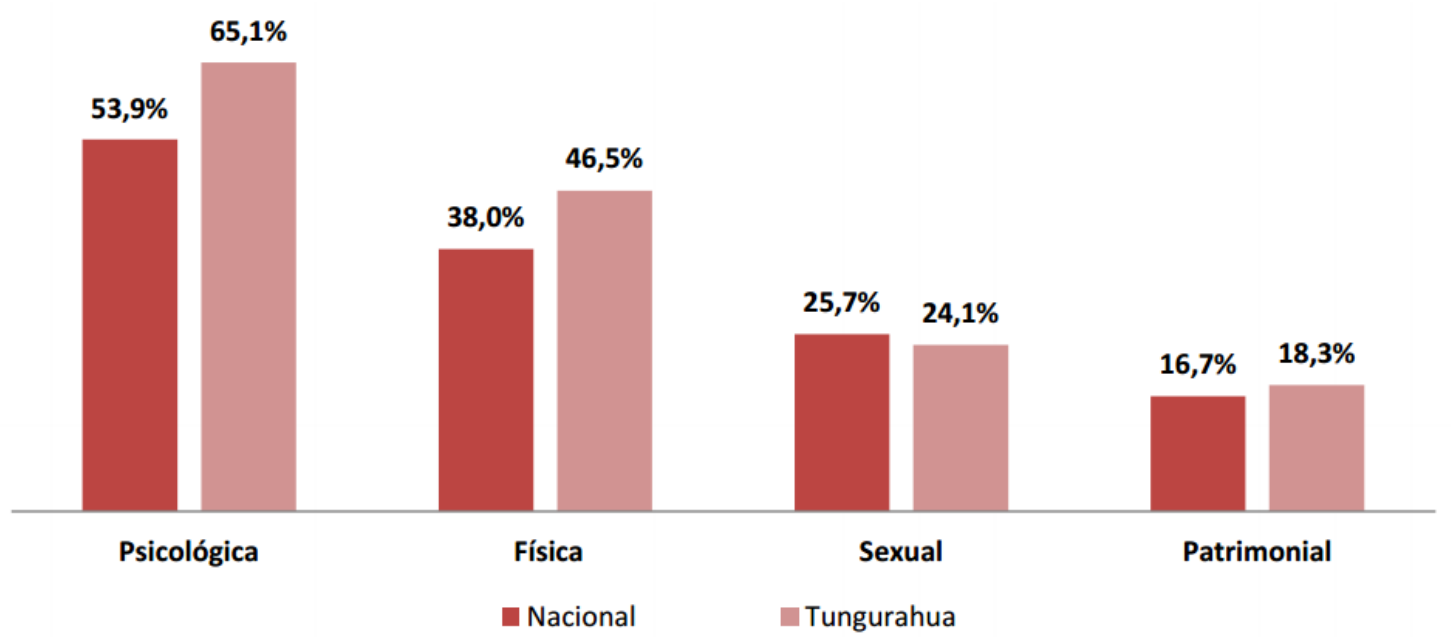

Gráfico 3. Mujeres que han vivido violencia psicológica, física, sexual y patrimonial - General.

Fuente: Primera Encuesta Nacional de Relaciones Familiares y Violencia de Género contra las Mujeres - Tungurahua.

De acuerdo a la encuesta en Tungurahua la violencia psicológica es la forma más recurrente de violencia de género con el $65,1 \%$., incluso a nivel nacional, seguida por la violencia física, la sexual y por último la patrimonial.

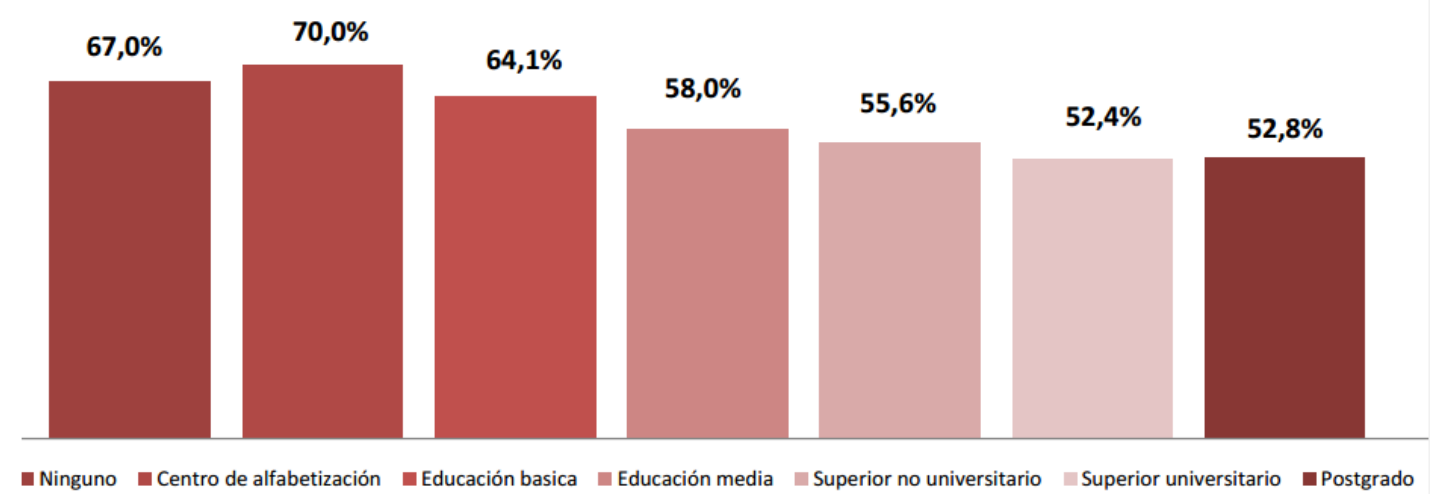

Grafico 4. Mujeres que han vivido algún tipo de violencia de género por nivel de instrucción.

Fuente: Primera Encuesta Nacional de Relaciones Familiares y Violencia de Género contra las Mujeres - Tungurahua.

Se puede establecer que en todos los niveles de instrucción la violencia de género sobrepasa el $50 \%$, sin embargo en las mujeres que tienen menos nivel de instrucción la violencia llega al $70 \%$, lo que indica que la problemática de violencia no solo 
Córdova P., L. V.; Córdova A., V. H., y Gómez A., H. F. El principio Pro Homine como base para la legislación de medidas de protección de género

afecta a un grupo determinado y menos aún se rige únicamente a personas con poca educación, sino por el contrario, afecta a cualquier persona sin contar con su nivel de preparación académica.

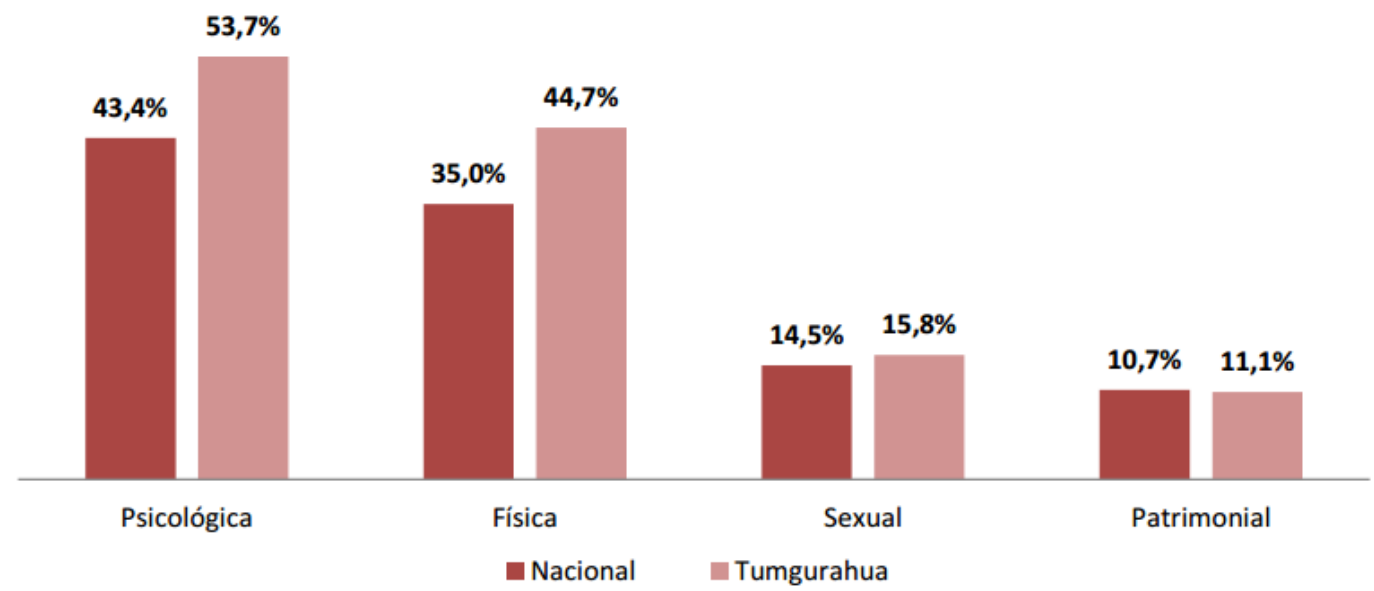

Grafico 5. Mujeres que han vivido violencia física, psicológica, sexual y patrimonial por su pareja o ex parejas.

Fuente: Primera Encuesta Nacional de Relaciones Familiares y Violencia de Género contra las Mujeres - Tungurahua.

El $53,7 \%$ de mujeres de Tungurahua ha vivido violencia psicológica en sus relaciones de pareja, es decir más de la mitad, a su vez en índice de violencia física en la provincia es muy alto, el mismo que casi alcanza a la mitad de las mujeres de la provincia.

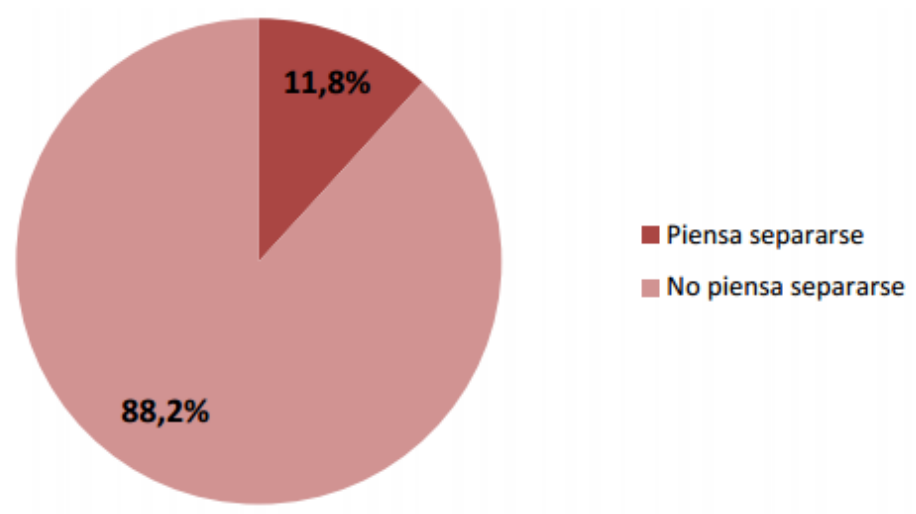

Grafico 6. Mujeres que han sufrido violencia de género, según su decisión con respecto a su pareja.

Fuente: Primera Encuesta Nacional de Relaciones Familiares y Violencia de Género contra las Mujeres - Tungurahua.

Al contrario de lo que se pensaría, la mayoría de las mujeres que han sufrido o sufren algún tipo de violencia, no piensan en separarse, en cambio solo un mínimo porcentaje lo considera. 
Córdova P., L. V.; Córdova A., V. H., y Gómez A., H. F. El principio Pro Homine como base para la legislación de medidas de protección de género

De acuerdo a lo establecido, la violencia de género e intrafamiliar en un problema de gran impacto en la sociedad y en su mayoría dentro de nuestra provincia, es claro que la cultura y las costumbres, han establecido que en general la violencia es algo normal dentro del desarrollo de una pareja o de una familia, lo que impide que esta problemática tenga fin, ya que son las propias víctimas quienes se niegan a dar fin a ese tipo de vínculos.

\subsection{Parámetros fundamentales para la legislación y juzgamiento en materia de violencia contra la mujer}

La tipificación de la violencia establece un adelanto importante en cuanto a la garantía de derechos, lo que demuestra el compromiso que tienen los Estados y la comunidad internacional por erradicar este problema. De acuerdo a lo que indica Fries \& Hurtado:

No hubo mucho debate respecto de donde alojar jurídicamente el tratamiento de la violencia, ya que cualquier otro fuero que no fuese el penal tendía a desvalorizar la violencia de género frente al tratamiento de otras violencias como las cometidas por un extraño y referidas fundamentalmente a homicidio, lesiones y delitos sexuales. También se asumió la necesidad de que fuera un tipo penal (en el código o en una ley especial) y no subsumido en los tipos delictuales existentes que no habían recogido históricamente esta problemática. (Fries \& Hurtado, 2010, p. 16).

Al hablar de la tipificación de la violencia este debía ser enmarcado dentro de un tipo penal, ya que la violación de derechos que esto implicaba, se encuentra al mismo nivel de otros delitos que violentan derechos fundamentales de las personas. A su vez su tipificación debería ser particular y no encajarla dentro de otros tipos de delitos normados anteriormente, ya que su tipificación particular, incluso en crear una ley especial, demuestra la importancia que se da a este problema.

\section{METODOLOGÍA}

La presente investigación utilizó el paradigma (cualitativo cuantitativo), el mismo que tuvo la modalidad bibliográfica-documental y de la modalidad de campo, en la modalidad bibliográfica-documental mediante a la utilización de artículos académicos, libros, doctrina, revistas, tesis, legislaciones, etc., que fueron la fuente de ayuda para la recolección de información frente al tema de investigación, además se aplicó la información receptada en base a documentos válidos y confiables a manera de información primaria, gracias a la cual pudimos determinar cuáles son las medidas de protección aplicada a las víctimas de violencia contra la mujer o miembros del núcleo familiar y a su vez conocer acerca del Principio Pro Homine.

Refiriéndonos a la modalidad de campo podemos determinar que se acudió en calidad de investigadores a distintas instituciones con la finalidad de obtener información acerca de la temática de investigación, ello nos ha permitido evaluar la situación real de la problemática planteada. 
Córdova P., L. V.; Córdova A., V. H., y Gómez A., H. F. El principio Pro Homine como base para la legislación de medidas de protección de género

\subsection{Método general}

El método general aplicado a la investigación fue el Inductivo, pues permitió analizar una serie de hechos y acontecimientos de carácter particular para llegar a generalidades que sirvan como referente en la investigación; con lo cual se puede establecer una conclusión general que afecta a la generalidad de casos.

\subsection{Método específico}

El método específico que ha sido utilizado en esta presente investigación ha sido el método analítico, ya que la investigación se ha descompuesto en todos sus elementos.

\subsection{Técnicas e instrumentos de recolección de información}

Dentro de la técnica utilizada en esta investigación se realizó el levantamiento de información sobre la temática, en la Fiscalía Provincial de Tungurahua; así también se realizó entrevista a los Jueces de Primera Instancia de la Unidad Judicial Penal con sede en el Cantón Ambato.

La técnica fundamental que se aplicó es la entrevista estructurada para captar la información de expertos y de personas relacionadas con la investigación para poder plasmar sus criterios y puntos de vista relacionada con la temática.

\section{EXPERIMENTACIÓN}

Para dar cumplimiento al primer objetivo de la investigación, correspondiente a diagnosticar la situación de las medidas de protección en los delitos de violencia contra la Mujer y miembros del núcleo familiar, se realizó el levantamiento de información relativo al mismo en la Fiscalía Provincial de Tungurahua donde se tomó indicadores relativos a la temática, en donde se desprende los siguientes resultados:

Tabla 2. Porcentaje de denuncias relacionadas a delitos de violencia contra la mujer o miembros del núcleo familiar en la Fiscalía de Tungurahua, enero- junio 2015.

\begin{tabular}{|l|c|c|}
\hline \multicolumn{1}{|c|}{ Delito } & Número De Causas & $\begin{array}{c}\text { Porcentaje de la } \\
\text { Totalidad De Delitos }\end{array}$ \\
\hline $\begin{array}{l}\text { Violencia Psicológica contra la } \\
\text { Mujer o miembros del núcleo } \\
\text { familiar }\end{array}$ & 941 & $14,19 \%$ \\
\hline $\begin{array}{l}\text { Violencia Física contra la Mujer } \\
\text { o miembros del núcleo familiar }\end{array}$ & 86 & $1,30 \%$ \\
\hline $\begin{array}{l}\text { Violencia Física contra la Mujer } \\
\text { o miembros del núcleo } \\
\text { familiar, si como resultado } \\
\text { produce daño, enfermedad o } \\
\text { incapacidad de 4 a 8 días }\end{array}$ & 30 & $0,45 \%$ \\
\hline $\begin{array}{l}\text { Violencia Física contra la Mujer } \\
\text { o miembros del núcleo familiar }\end{array}$ & 18 & $0,27 \%$ \\
\hline
\end{tabular}


Córdova P., L. V.; Córdova A., V. H., y Gómez A., H. F. El principio Pro Homine como base para la legislación de medidas de protección de género

si como resultado produce

daño, enfermedad o

incapacidad de 9 a 30 días

Fuente: Datos Fiscalía de Tungurahua.

Para dar cumplimiento con el segundo y cuarto objetivo de la investigación que son establecer las medidas de protección aplicables a las víctimas de delitos de violencia contra la mujer o miembros del núcleo familiar y contrastar las medidas de protección de las víctimas de delitos de violencia contra la mujer o miembros del núcleo familiar en relación al Principio Constitucional Pro Homine, se realizó entrevistas a Jueces y Juezas de la Unidad de Garantías Penales con sede en el Cantón Ambato en Tungurahua.

Tabla 3. Jueces entrevistados.

\begin{tabular}{|l|l|}
\hline \multicolumn{1}{|c|}{ Jueces y Juezas de la Unidad de Garantías Penales con sede en el Cantón } \\
Ambato en Tungurahua
\end{tabular}

Fuente: Unidad Judicial de Garantías Penales.

\section{ANÁLISIS DE ENTREVISTAS Y LEVANTAMIENTO DE INFORMACIÓN}

\subsection{Levantamiento de Información}

Dentro de la información que se otorgó por parte de la Dra. Adelaida Palate, de la totalidad de causas que se tramitan dentro de la Fiscalía Provincial de Tungurahua, el delito que se tramita en mayor porcentaje dentro de esta institución es el de Violencia Psicológica contra la mujer o miembros del núcleo familiar, en comparación a este no existe un índice igual de alto en delitos de violencia física contra este grupo específico, pero dentro de ambos delitos se da la necesidad de emisión de medidas de protección lo cual no se realiza de manera inmediata o no se da requerimiento a las mismas.

\subsection{Entrevista Jueces}

\subsubsection{Juez 1}

Dentro de la entrevista con la jueza supo manifestar que la medida de protección más aplicada a víctimas de violencia contra la mujer o miembros del núcleo familiar es la extensión de una boleta de auxilio. 
A su vez en cuanto al conocimiento de lo que implica el Principio Constitucional Pro Homine, estableció que este principio es una forma de interpretación jurídica la cual debe buscar el beneficio para el ser humano, en aplicación concreta al delito de violencia contra la mujer o miembros del núcleo familiar se hablaría de la interpretación de la norma en favor de las víctimas de estos delitos, quienes están siendo vulnerados en sus derechos, este principio es garantizado tanto por normas nacionales como internacionales.

Los criterio en los cuales se fundamenta la emisión de medidas de protección es la protección y a su vez la prevención de cualquier vulneración que pueda sufrir la persona, es decir estas medidas se emiten con el propósito de cuidar la integridad de la persona, en cuanto a la efectividad de las mismas supo establecer que las mismas si son efectivas, por cuanto las medidas que se expide mantienen alejado al agresor de la víctima, lo cual cuida la integridad de la misma. Para finalizar la entrevistada expresa que para poder mejorar el sistema en cuanto a medidas de protección a víctimas de violencia contra la mujer o miembros del núcleo familia, se debe dar la promulgación de la norma en este caso el (Código Orgánico Integral Penal, Registro Oficial: No 180, 2014), que como norma es efectiva en la protección de las víctimas de este tipo de delitos.

\subsubsection{Juez 2}

Dentro de la entrevista realizada al juez, en cuanto a las medidas más aplicada a las víctimas de violencia contra la mujer o miembros del núcleo familiar estableció que dentro de nuestra circunscripción como cantón Ambato, las más aplicadas son la emisión de boleta de auxilio, la prohibición de acercarse del denunciado a la víctima o a su vez se ordena la salida del agresor en casos en los cuales se comparta vivienda con la víctima de violencia. En relación al conocimiento del entrevistado acerca del Principio Constitucional Pro Homine, el mismo indica que dentro de nuestra constitución se encuentra dispuesto en el artículo 11 numeral 5, que expresa que la norma se debe aplicar en forma que sea más favorable a su efectiva vigencia, en este caso el derecho de las personas, por lo cual como jueces está dentro de sus obligaciones en la administración de justicia tomar en cuenta a este principio de aplicación.

En cuanto a los criterios que se consideran para la emisión de medidas de protección, indica que se debe analizar el caso en concreto y a su vez la fundamentación que establezca el titular de petición, que sería Fiscalía, con lo cual se busca cesar la agresión o prevenir que exista una nueva agresión.

A su vez en cuanto a la efectividad de las mismas, establece que a partir de la promulgación del (Código Orgánico Integral Penal, Registro Oficial: No 180, 2014), la efectividad de las medidas de protección a incrementado, ya que todo tipo de violencia se tipifico como delito dentro de este Código dándole más relevancia a las víctimas de este tipo de hechos, a su vez desde la vigencia del (Código Orgánico Integral Penal, Registro Oficial: No 180, 2014) se encuentra tipificado el incumplimiento de medidas de protección, lo que da una mayor garantía de 
Córdova P., L. V.; Córdova A., V. H., y Gómez A., H. F. El principio Pro Homine como base para la legislación de medidas de protección de género

protección a quien tiene a su favor este tipo de medidas, esto en comparación con tipos anteriores que se daba caso omiso a la disposición de estas medidas.

En cuanto a la forma en que se puede mejorar el sistema de medidas de protección en el ámbito judicial, el entrevistado establece que aunque los cambios han sido positivos, no se puede hablar de efectividad al cien por ciento, por ello el Estado debería crear un organismo independiente el cual puede tratar esta problemática, como se establece en normativas de otros países, que tienen como objetivo el dar un seguimiento a partir de la emisión de la medida, ya que el Estado a su vez también busca conservar y amparar al núcleo familiar, que se puede ver afectado por estad medidas, por lo cual es importante no solo emitir las medidas sino valorar que efectividad tiene la misma y a su vez buscar la regeneración del núcleo familiar por medio de un seguimiento por parte de peritos de diferentes áreas pertenecientes a este sistema de antelación, como complemento a lo expuesto el entrevistado recalca que este tipo de delitos se dan en todo tipo de hogar, sin importar índole social o cultural.

\subsubsection{Juez 3}

En esta entrevista el señor Juez supo manifestar que las medidas más aplicadas a víctimas de violencia contra la mujer o miembros del núcleo familiar son primeramente la boleta de auxilio, a su vez es solicitada con frecuencia la prohibición de acercamiento del agresor a la víctima, esto en relación a su vez a la prohibición de hacer actos de persecución o intimidación hacia la víctima y la salida del agresor del domicilio en el que convive con la víctima.

En cuanto al conocimiento del principio Pro Homine nos establece que este principio tiene como objetivo el buscar el bienestar de la persona, en relación a las medidas de protección esto implica que las mismas dan protección a cualquier miembro de la familia, no necesariamente la mujer, es decir que la emisión de estas medidas de protección buscar salvaguardar la integridad de la persona en general. En cuanto a los criterios que se toman para otorgar las medidas de protección, señala que ellos como jueces para emitir medidas de protección deben considerar la fundamentación que es enviada por Fiscalía y no solamente una fundamentación en derecho sino también las circunstancias del caso concreto, en base a esto ellos tienen la potestad de emitir o no dichas medidas, ya que a criterio del entrevistado estas pueden ser mal utilizadas, por ellos la efectividad de las mismas que a criterio del entrevistado lo son, pueden ser perjudiciales en ciertos casos. Y en cuanto a cómo mejorar la administración de estas, indica que debe existir mayor control al momento de la emisión de estas ya que deben tener una fundamentación valida, es decir que se constate la necesidad de estas medidas, para prevenir el mal uso de las mismas.

\subsubsection{Juez 4}

Las medidas de protección más aplicadas son la boleta de auxilio en favor de la víctima, en primer plano, la segunda la prohibición de acercarse a la víctima y 
después la prohibición de hacer actos de intimidación ya sea por sí o por medio de otras personas y dándose el caso que la peligrosidad del agresor sea muy alta se dispone la salida del mismo del lugar donde comparte la vivienda con la víctima. Acerca del principio Pro Homine, establece que este nace a partir de los derechos humanos y se encuentra ligado a la persona, que tiene como objetivo tutelar los derechos de la persona como prima facie, es decir a primera vista ante el juzgador. En cuanto a este principio con relación a las extensión de medidas de protección, el juzgador debe verificar la existencia de méritos, los cuales son remitidos por Fiscalía, de allí el análisis que realiza el juzgador toma en cuenta este principio, en este caso en beneficio de las víctimas, es decir que es obligación de juzgador tomar en cuenta las mismas.

A criterio del entrevistado si existe efectividad en las medidas de protección, pero tomando en cuenta que la efectividad de las mismas se ve influenciada en el desempeño de otros factores como la respuesta inmediata por parte de los servidores policiales, otro factor que recalca el entrevistado es el avance que se ha tenido al tipificar como delito el incumplimiento de estas medidas a partir de la promulgación del COIP, lo que ha beneficiado a disminuir este tipo de agresiones. En cuanto a la mejora que puede existir en cuestión de medidas de protección, indica que eso abarca a diferentes instituciones, primero a la Fiscalía ya que el desconocimiento acerca de la temática de violencia, puede tomar como una denuncia a cuestiones muy superficiales lo cual no aportaría nada a el proceso en sí, otro aspecto importante es la notificación al agresor, con ello no se viola el derecho de la otra parte, en cuanto a la mejora en la efectividad de las medidas en sí, se indica que puede establecerse políticas públicas en cuanto a capacitación sobre la temática no solo a profesionales, sino niños, adolescentes, etc.; para dar a conocer derechos y medios por los cuales hacer respetar los mismos en el caso de violencia, ya que muchas veces la víctima se retracta de la denuncia interpuesta.

\subsection{Análisis General}

De acuerdo con la información recopilada es claro que al tipificar la violencia contra la mujer o miembros del núcleo familiar, los índices de estas denuncias abarcan la gran mayoría de los procesos que se tramitan dentro de Fiscalía, por tanto esto incide directamente en la celeridad con la que estos son tramitados, a su vez el cambio de legislación tuvo un cambio directo en el proceso de estos delitos, que significo que las medidas de protección no sean de inmediata disposición, por tanto las víctimas de este tipo de delitos quedan en un estado de vulnerabilidad, en relación a esto los entrevistados establecen que es necesario que se establezcan fundamentos para que el juez o jueza considere si estas son necesarias o no, ya que pueden afectar a la parte denunciada, pero se deja de lado a la integridad de la víctima y existe hasta cierto punto una contradicción, ya que los jueces y juezas indicaron que este principio es de gran importancia y se encuentra ligado a los derechos humanos, es decir que el juzgador debe asegurar la integridad de la víctima en cualquier circunstancia, lo que se ha violado a partir de este cambio. Es importante reconocer que existe una diversidad de medidas que pueden ser 
Córdova P., L. V.; Córdova A., V. H., y Gómez A., H. F. El principio Pro Homine como base para la legislación de medidas de protección de género

aplicadas, tanto antes, durante y después del proceso, con lo cual se busca cuidar la integridad de la víctima sin afectar al procesado.

\section{CONCLUSIONES}

A partir del cambio de legislación en materia de violencia contra la mujer o miembros del núcleo familiar, se dio un cambio en cuanto al procedimiento en esta temática. Es claro que las medidas de protección han sido y son una garantía de seguridad para la víctima, e claro que existe una problemática al momento de la solicitud de las mismas por el alto número de causas que se tramitan dentro de la Fiscalía. Es decir que no existe una correcta emisión de estas medidas, ni tampoco se da importancia o prioridad a este tema, argumentando que dentro de esta institución existen delitos más importantes que gestionar, lo que pone en riesgo la integridad de quien establece la denuncia, al no recibir una garantía inmediata como se daba con la ley anterior.

1. Las medidas de protección aplicables a los casos de violencia contra la mujer o miembros del núcleo familiar, se encuentran establecidas dentro del (Código Orgánico Integral Penal, Registro Oficial: No 180, 2014) en su artículo 558. Estas medidas no han cambiado en comparación a las establecidas dentro de la Ley 103. Al hablar de delitos de violencia contra la mujer o miembros del núcleo familiar, se especifican ciertas medidas de protección, las cuales buscan proteger la integridad de la víctima, las mismas que en orden de mayor uso son: Extensión de una boleta de auxilio a favor de la víctima o de miembros del núcleo familiar en el caso de violencia contra la mujer o miembros del núcleo familiar, prohibición a la persona procesada de acercarse a la víctima, testigos y a determinadas personas, en cualquier lugar donde se encuentren, prohibición a la persona procesada de realizar actos de persecución o de intimidación a la víctima o a miembros del núcleo familiar por sí mismo o a través de terceros y orden de salida de la persona procesada de la vivienda o morada, si la convivencia implica un riesgo para la seguridad física, psíquica o sexual de la víctima o testigo, ya que tienen como premisa el cesar la violencia existente y prevenir un delito más grave. Es claro que las medidas deben ser emitidas de acuerdo al caso concreto, pero debería existir mayor aplicación de medidas de protección como el ordenar el tratamiento respectivo al que deben someterse la persona procesada o la víctima y sus hijos, ya que es una medida que no es utilizada, pero en sí, sería la más efectiva en este tipo de delitos, buscando así una estabilidad tanto para la víctima, como el agresor y su entorno familiar. El Principio Constitucional Pro Homine, es un criterio interpretativo que informa todo el derecho de los derechos humanos, en virtud del cual se debe acudir a la norma más amplia, o a la interpretación más extensiva, cuando se trata de reconocer derechos protegidos. Este principio es y debe ser un importante instrumento para el juzgador, ya que la protección de los derechos de la mujer se encuentran plasmados dentro de normativa internacional, así como los lineamientos para el trámite. Este principio también puede manifestarse o ser aplicado por el resto de operadores jurídicos. Sin lugar a duda, es un principio que 
Córdova P., L. V.; Córdova A., V. H., y Gómez A., H. F. El principio Pro Homine como base para la legislación de medidas de protección de género

debe ser observador a su vez por el legislador a fin de no crear normas regresivas-limitantes de la protección y vigencia de los derechos humanos. Las medidas de protección son disposiciones y ordenes creados para proteger la seguridad de las personas, a fin de hacer efectivo el cuidado y protección de la víctima de la agresión, con respecto a la agresión misma y a su agresor; son mecanismos que buscan brindar apoyo y protección a las víctimas de las agresiones e impedir la continuación de estas, a su vez el principio Pro Homine enseña el fundamento para interpretar los derechos fundamentales y a su vez tiene un sentido protector, que debe adjudicarse la interpretación a favor del más débil y como debe dirimirse una decisión jurisdiccional, se debe dar la solución más beneficiosa a los derechos del individuo.

Este principio indica que el juez debe seleccionar y aplicar la norma que, resulte más favorable a la persona humana, para su libertad y ejercicio de sus derechos, es decir que el juzgador al momento de encontrarse frente a una solicitud de medidas de protección este debe verificar la existencia de méritos, los cuales son remitidos por Fiscalía, de allí el análisis que realiza el juzgador toma en cuenta este principio, en este caso en beneficio de las víctimas, es decir que es obligación de juzgador tomar en cuenta las mismas, ya que se pone en juego la integridad y seguridad de la persona víctima de violencia, esto de manera inmediata y eficaz como versa dentro de la Convención Interamericana para prevenir, sancionar y erradicar la violencia contra la Mujer. En base a los parámetros fundamentales para la legislación y juzgamiento en materia de violencia contra la mujer que ha establecido las Naciones Unidas, se ha cumplido muchos de ellos pero a su vez existen algunas deficiencias las cuales impiden un procedimiento óptimo para este tipo de delitos.

\section{REFERENCIAS}

Ardrey, R. (1966). The Territorial Imperative: A Personal Inquiry into the Animal Origins of Property and Nations. New York: Atheneum.

Banchs, M. (1966). Violencia de Género. Revista Venezolana de Análisis de Coyuntura, 2, 15. Recuperado de http://www.ucv.ve/fileadmin/user upload/faces/iies/ANALISIS DE COYUNTURA VOLUMEN II No 2 JULIO DICIEMBRE 1996.pdf\#page $=15$

Boulding, E. (2010). La violencia y sus causas: Las mujeres y la violencia social. Paris: UNESCO.

Bunch, C. (1991). Los Derechos de la Mujer como Derechos Humanos: Mujer y Violencia Doméstica. Santiago de Chile: Instituto de la Mujer.

Cabanellas, G. (2003). Diccionario Jurídico Elemental. Heliasta S.R.L. 
Córdova P., L. V.; Córdova A., V. H., y Gómez A., H. F. El principio Pro Homine como base para la legislación de medidas de protección de género

Castilla, K. (2009). El principio pro persona en la administración de justicia. Cuestiones constitucionales, 20, 65-83. Recuperado de http://www.scielo.org.mx/scielo.php?script=sci arttext\&pid=S140591932009000100002\&lng $=$ es\&

Código Orgánico Integral Penal, Registro Oficial № 180. Quito. (10 de Febrero de 2014).

Comisión Andina de Juristas (2003). Los derechos humanos y la Globalización: avances y retrocesos. Lima, Perú: CAJ.

Constitución de la República del Ecuador, Registro Oficial № 242 del 20 de Octubre del 2008. (s.f.).

Constitución Política de la República del Ecuador, Registro Oficial No 27818 de marzo de 1998. (s.f.)

Convención Interamericana de Belem do Para, B.O. (9 de Abril de 1996).

Corsi, J. (2006). Maltratos y abuso en el ámbito doméstico. Quito, Ecuador: Patronato San José.

Departamento de Asuntos Económicos y Sociales - División para el Adelanto de la Mujer (2010). Manual de legislación sobre la violencia contra la mujer. Nueva York: Naciones Unidas.

Díaz, A. (2009). La efectividad de las medidas de protección frente a la violencia familiar. Recuperado de https://trabajadorjudicial.wordpress.com/la-efectividadde-las-medidas-de-proteccion-frente-a-la-violencia-familiar/

Diccionario RAE - Real Academia Española (2001). Diccionario de la lengua española. Madrid: Espasa Calpe.

Fries, L., \& Hurtado, V. (2010). Estudio de la información sobre la violencia contra la mujer en América Latina y el Caribe. Chile, Santiago de Chile: Naciones Unidas.

Galarza, M. (2010). La falta de aplicación de las Medidas de Amparo dictadas por la Comisaría de la Mujer y la Familia dentro de las acciones legales, en el primer semestre del año 2009, provoca el incremento de violencia intrafamiliar en el cantón Ambato. Provincia de Tunguragua.

Gutiérrez, F. (2005). Manual de Aplicación de Normas Internacionales de Derechos Humanos en el Ámbito Jurídico Ecuatoriano. Guayaquil, Ecuador: Impresos Anabel.

Henderson, H. (2004). Los Tratados Internacionales de Derechos Humanos en el orden interno: la importancia del principio Pro Homine. Revista IIDH. 39(29). 
Córdova P., L. V.; Córdova A., V. H., y Gómez A., H. F. El principio Pro Homine como base para la legislación de medidas de protección de género

Recuperado de http://angelduran.com/docs/Cursos/CCDC2013/mod02/02-023 L3Henderson.pdf

Instituto Nacional de Estadísticas y Censos (2011). Primera Encuesta Nacional de Relaciones Familiares y Violencia de Género contra las Mujeres Tungurahua. Recuperado de http://www.ecuadorencifras.gob.ec/violencia-de-genero/

Laurenzo, P. (2005). La violencia de género en la Ley Integral Valoración políticocriminal. Revista Electrónica de Ciencia Penal y Criminología, 07-08, 08:1-08:23. Recuperado de http://criminet.ugr.es/recpc/07/recpc07-08.pdf

Lorenz, K. (2002). On Agression. Londres: Routledge.

Ojeda, L. (2010). Violencia, delincuencia e inseguridad en el Ecuador. Quito: UNAP.

Unidas, C. F. (1945). Estados Unidos: San Francisco.

Walker, L. (1979). The Battered Women. Nueva York: Harper and Row Publishers.

\section{AUTORES}

\section{Laura Victoria Córdova Pérez}

Abogada - Pontificia Universidad Católica del Ecuador, Sede Ambato. Abogada adjunta en libre ejercicio profesional para el Estudio de Asesoría Legal "ESDALEX", abogada en libre ejercicio, miembro de Jóvenes UNASUR Ecuador.

laura.cordova@uasb.edu.ec

\section{Víctor Hugo Córdova Aldás}

Ingeniero de empresas, doctor por la Universidad Rey Juan Carlos, España. Profesor - investigador Universidad Técnica de Ambato. Coordinador de posgrados FCADM UTA. Docente - tutor en programas de maestría UTA.

victorhcordova@uta.edu.ec

cordovav2@yahoo.es

\section{Héctor Fernando Gómez Alvarado}

Ingeniero en Informática, doctor por la Universidad Nacional de Educación a Distancia UNED, España. Profesor - investigador Universidad Técnica de Ambato. director de posgrados - UTA. Docente - tutor en programas de maestría UTA. hf.gomez@uta.edu.ec

hfgomez6@gmail.com 